Edith Cowan University

Research Online

ECU Publications Post 2013

2015

Compliance to exercise-oncology guidelines in prostate cancer survivors and associations with psychological distress, unmet supportive care needs, and quality of life

\author{
Daniel A. Galvao \\ Edith Cowan University \\ Robert U. Newton \\ Edith Cowan University \\ Robert A. Gardiner \\ Edith Cowan University \\ Afaf Grigis \\ Steven J. Lepore
}

See next page for additional authors

Follow this and additional works at: https://ro.ecu.edu.au/ecuworkspost2013

Part of the Health Psychology Commons, and the Oncology Commons

10.1002/pon.3882

This is an Author's Accepted Manuscript of: Galvão, D.A., Newton, R.U., Gardiner, R.A., Girgis, A., Lepore, S.J., Stiller, A., Mihalopolous, C., Occhipinti, S., Chambers, S.K. (2015). Compliance to exercise-oncology guidelines in prostate cancer survivors and associations with psychological distress, unmet supportive care needs, and quality of life in Psycho-Oncology, 24(10), 1241-1249. Available here.

This Journal Article is posted at Research Online.

https://ro.ecu.edu.au/ecuworkspost2013/1225 


\section{Authors}

Daniel A. Galvao, Robert U. Newton, Robert A. Gardiner, Afaf Grigis, Steven J. Lepore, Anna Stiller, Cathrine Mihalopolous, Steven Occhipinti, and Suzanne K. Chambers 


\title{
Compliance to exercise-oncology guidelines in prostate cancer survivors and associations with psychological distress, unmet supportive care needs, and quality of life
}

\author{
Daniel A. Galvão', Robert U. Newton', Robert A. Gardiner 1,2,3, Afaf Girgis ${ }^{4}$, Stephen J. Lepore ${ }^{5}$, Anna Stiller ${ }^{6}$, \\ Cathrine Mihalopolous ${ }^{7}$, Stefano Occhipinti ${ }^{8}$ and Suzanne K. Chambers $1,2,6,8,9 *$ \\ 'Edith Cowan University Health and Wellness Institute, Edith Cowan University, Perth, Australia \\ ${ }^{2}$ University of Queensland Centre for Clinical Research, University of Queensland, Brisbane, Australia \\ ${ }^{3}$ Department of Urology, Royal Brisbane and Women's Hospital, Brisbane, Australia \\ ${ }^{4}$ Psycho-Oncology Research Group, Ingham Institute for Applied Medical Research, South Western Sydney Clinical School, University of NSW, Sydney, \\ Australia \\ ${ }^{5}$ Department of Public Health, Temple University, Philadelphia, USA \\ ${ }^{6}$ Cancer Council Queensland, Brisbane, Australia \\ ${ }^{7}$ Deakin Health Economics, Deakin University, Melbourne, Australia \\ ${ }^{8}$ Menzies Health Institute of Queensland, Griffith University, Brisbane, Australia \\ ${ }^{9}$ Prostate Cancer Foundation of Australia, Sydney, Australia
}

*Correspondence to: Menzies Health Institute of Queensland, Griffith University, Brisbane, Australia.

E-mail: suzanne.chambers@ griffith.edu.au

Trial registration: ACTRN / $26 / 1000392965$

Received: 15 October 2014

Revised: 7 April 2015

Accepted: 20 May 2015

\begin{abstract}
Objective: The purpose of this study was to determine prevalence of Australian prostate cancer survivors meeting contemporary exercise-oncology guidelines and identify associations with distress, unmet supportive care needs, and quality of life.

Methods: A population-based cohort of 463 prostate cancer survivors who were on 10.8 months post-curative therapy was assessed for compliance with current exercise guidelines for cancer survivors, motivational readiness for physical activity, psychological distress, unmet supportive care needs, and quality of life.

Results: Only 57 men $(12.3 \%)$ reported sufficient exercise levels (150 min of moderate intensity or $75 \mathrm{~min}$ of strenuous exercise per week and twice weekly resistance exercise), $186(\mathbf{4 0 . 2 \%})$ were insufficiently active, and $220(47.5 \%)$ were inactive. Among inactive men, 99 (45.0\%) were in the contemplation or preparation stage of motivation readiness. Inactive men had higher global distress $(p=0.01)$ and Brief Symptom Inventory-Anxiety $(p<0.05)$ than those who were insufficiently active. Total Supportive Care Needs and International Prostate Cancer Symptom scores were higher in inactive than insufficiently and sufficiently active men $(p<0.05)$. Lack of physical activity contributed to poorer quality of life.

Conclusions: Only a small proportion of Australian prostate cancer survivors met contemporary exercise-oncology recommendations despite increasing recognition of exercise to improve patient outcomes. Strategies are urgently required to increase prostate cancer survivors' participation in aerobic and resistance exercise training.

Copyright @ 2015 John Wiley \& Sons, Ltd.
\end{abstract}

\section{Background}

In the past decade, several exercise trials have been conducted with prostate cancer survivors mainly in the setting of localized disease during or following radiation and androgen deprivation therapy [1-8]. Overall, consistent positive outcomes have been reported across studies strongly indicating that both resistance and aerobic exercises is beneficial in reducing a number of treatment-related toxicities and improving symptoms. Furthermore, regular physical activity has been associated with lower incidence of prostate cancer death with those undertaking $\geq 3 \mathrm{~h}$ per week of vigorous activity having $\sim 60 \%$ lower risk of prostate cancer death compared with men undertaking $<1 \mathrm{~h}$ per week of vigorous activity [9]. This finding adds to the growing body of evidence suggesting that exercise may extend survival for cancer patients $[10,11]$.

As a result of recent advancements in the field of exercise oncology, the American Cancer Society and American College of Sports Medicine [12,13] published exercise guidelines for cancer survivors including prostate cancer survivors. Survivors are advised to avoid inactivity regardless of cancer type or stage, even when undergoing difficult treatments; undertake 150 min per week of moderate or $75 \mathrm{~min}$ per week of vigorous aerobic exercise or an equivalent combination; and perform resistance 
exercise of moderate or high intensity on two or more days per week, the latter being particularly important for prostate cancer survivors who experience musculoskeletal toxicities. It is unclear how well promoted these guidelines are, and these may vary according to regions, hospitals, and treating oncologists/urologists.

It is unknown if prostate cancer survivors are meeting these recommendations and importantly undertaking the contemporary prescription of both aerobic and resistance exercise. The purpose of this study was to determine the prevalence of adherence to current exercise-oncology guidelines in Australian prostate cancer survivors and evaluate associations with psychological distress, unmet supportive care needs, and quality of life (QoL). We hypothesized that few Australian prostate cancer survivors are meeting current recommendations of exercise guidelines and that those meeting recommendations would have less distress and unmet supportive care needs and better QoL.

\section{Methods}

\section{Participants}

Eligible participants were men with localized prostate cancer in Queensland after 1 January 2011 recruited through the Queensland Cancer Registry [14]. Inclusion criteria were to have undergone/be undergoing prostate cancer treatment; able to read and speak English; no previous history of head injury, dementia, or current psychiatric illness; no concurrent cancer; and physician clearance. The study was approved by the Griffith University Human Research Ethics Committee as well as ethics committees of hospitals across Queensland, and all participants provided written informed consent. Participants were recruited as part of a randomized controlled trial that is ongoing [14], with cross-sectional baseline data reported in this paper.

\section{Materials}

Assessment was via computer-assisted telephone interview. Study variables were assessed using previously validated and reliable self-report measures [14].

\section{Physical activity}

Adherence to exercise was measured using the Godin Leisure-Time Exercise Questionnaire, which assesses the average frequency and duration of mild, moderate, strenuous, and resistance exercises during free time in a typical week in the past month [15]. Mild exercise was activity that required minimal effort with examples such as fishing, golf, and easy walking. Moderate exercise was activity that was not exhausting such as fast walking, tennis, easy cycling, and easy swimming. Strenuous exercise included activity in which the heart beats rapidly such as running, jogging, playing football, vigorous swimming, and vigorous cycling. Resistance exercise was added to this scale and included examples such as lifting weights, push-ups, sit-ups, and using resistance bands. Definition of sufficient activity was based on recommended physical activity guidelines for people with cancer according to the American College of Sports Medicine and American Cancer Society [13], which is to accumulate $150 \mathrm{~min}$ of moderate-intensity or $75 \mathrm{~min}$ of vigorous (or a combination of moderate and vigorous) aerobic exercise per week, in addition to resistance exercise sessions twice weekly.

\section{Psychological distress and unmet supportive care needs}

The Brief Symptom Inventory-18 provided a global measure of current psychological distress with subscales of anxiety, depression, and somatization [16]. The sum of each of the three subscales comprises the Global Severity Index with higher scores indicating greater distress. The Supportive Care Needs Survey Short Form-34 assessed help required across psychological, health system and information, patient care and support, physical and daily living, and sexuality needs. An eight-item prostate cancer-specific module previously developed by our group was added [17]. Items are rated from 'No need/not applicable' to 'High need' with higher scores indicating greater unmet need.

\section{Quality of life}

The Assessment of QoL-8D (AQoL-8D) assessed healthrelated QoL [18], and the International Prostate Symptom Score (IPSS) [19] and symptom subscales of the Expanded University of California, Los Angeles Prostate Index Composite (EPIC) [20,21] measured disease-specific QoL.

\section{Motivational readiness for physical activity}

Motivational readiness for physical activity assessed intention to become more physically active across precontemplation, contemplation, preparation, action, and maintenance [22]. Respondents were assigned to one of the five stages of motivational readiness based on their most highly rated item. If there were more than one item that met these criteria, the participant was assigned to the stage that was at the highest in the stage of change process.

\section{Remoteness}

Suburb and postcode of participants' residence at diagnosis were assigned a Statistical Area 2 region using a concordance provided by the Australian Bureau of Statistics. Based on 2011 and 2012 data, areas were matched to the appropriate remoteness category: 'major city', 'inner regional'; 'outer regional'; 'remote and very remote'.

\section{Statistical methods}

Age and education were adjusted in all analyses. Due to the lack of independence and relationship between physical activity levels and a person's comorbidities and 
waist circumference, these latter two variables were not included as covariates. Analyses of covariance were conducted to assess whether the three physical activity groups differed in their scores on QoL, psychological distress, and supportive care needs controlling for possible confounders. All post hoc comparisons used the Tukey honest significant difference test, or the Dunnett's in cases where Levene's test of homogeneity of variance was significant. Prior to analyses, examination of frequencies, normal probability plots, and scatterplots revealed the bowel symptoms and bother to be significantly negatively skewed. Square root transformations were undertaken for these variables but led to no meaningful differences in results and hence were applied.

\section{Results}

\section{Participants}

Between September 2011 and November 2012, 1899 patients were identified as potentially eligible; of these, 1770 doctors were contacted for permission to contact their patients of whom 1564 (88.4\%) agreed; 1501 patients were sent letters inviting them to the study; of these, 679 (45.2\%) agreed to participate, and after screening for eligibility and consent, 463 agreed to proceed and completed the baseline assessment. Participants were on average 10.8 months post-diagnosis $(\mathrm{SD}=3.03$, range 0.6-21.6 months), and the majority (95.5\%) had already undergone or commenced treatment $(n=442$; Table 1$)$. Remoteness of residence was representative of the male population aged 43-89 years in Queensland during 2012 $(p=0.62)$, and of prostate cancer patients aged $43-89$ years diagnosed in Queensland during $2011(p=0.20)$.

\section{Compliance to exercise-oncology guidelines}

Of 463 prostate cancer survivors, only 57 participants (12.3\%) reported sufficient levels of exercise $(\geq 150 \mathrm{~min}$ of moderate-intensity exercise or $\geq 75$ min of vigorous exercise per week and two resistance sessions per week), $186(40.2 \%)$ were insufficiently active (not meeting recommendations), and $220(47.5 \%)$ were inactive (no moderate or vigorous activity). Among those who were insufficiently active, 95 (20.5\% of the total sample) met the aerobic but not resistance exercise guidelines.

Among those who were inactive, the mean weekly level of mild exercise reported was $157.9 \min (S D=252.3)$, with no moderate and strenuous exercise, and a mean of 0.4 sessions $(\mathrm{SD}=1.4)$ per week of resistance exercise. The insufficiently active group had a mean mild exercise level of $151.05 \mathrm{~min}(\mathrm{SD}=259.9)$, moderate exercise of $123.7 \mathrm{~min}(\mathrm{SD}=127.7)$, strenuous exercise of $46.3 \mathrm{~min}$ $(\mathrm{SD}=99.2)$, and resistance exercise of 0.4 sessions $(\mathrm{SD}=1.2)$ per week. The sufficiently active group had a mean mild exercise level of $195.7 \mathrm{~min}(\mathrm{SD}=340.0)$, moderate exercise of $248.4 \mathrm{~min}(\mathrm{SD}=214.7)$, strenuous exercise of $133.9 \mathrm{~min}(\mathrm{SD}=158.8)$, and resistance exercise of 4.3 sessions $(S D=2.2)$ per week.

In the total sample, the majority of participants, $54.6 \%$ $(n=253)$, indicated that they were in the maintenance stage of motivational readiness for physical activity, $10.4 \%(n=48)$ in the action stage, $19.2 \%(n=89)$ in preparation stage, $9.8 \%(n=45)$ in contemplation stage, and $3.9 \%(n=18)$ in the pre-contemplation stage. When comparing among the three physical activity groups, $45.0 \%$ $(n=99)$ of inactive men were in contemplation or preparation stage, compared with $18.3 \%$ of the insufficiently active men, and $0.02 \%$ of the active men. See Table 1 for further details. Using a division of lower (pre-contemplation, contemplation, and preparation) and higher motivational stages (action and maintenance), active men (insufficient/sufficient) more frequently reported a higher stage of motivational readiness, compared with inactive men $(p<0.001)$.

\section{Sociodemographic and clinical variables}

The age of participants differed in relation to compliance to exercise-oncology guidelines $(p<0.001)$. Inactive men were older than those insufficiently $(p<0.01)$ and sufficiently active $(p=0.001)$. Inactive men had greater mean waist circumference compared with insufficiently $(p<0.001)$ and sufficiently active men $(p=0.001)$. As well, inactive men had higher rates of arthritis $(p<0.05)$ and diabetes $(p<0.05)$ than active men (sufficient/insufficiently active). Furthermore, groups differed when comparing those with a tertiary or trade education with those with a lower educational level $(p<0.001)$. There were no differences for BMI $(p=0.09)$, time since diagnosis $(p=0.96)$, or time since treatment $(p=0.08)$.

\section{Psychological distress and unmet supportive care needs}

Inactive men had higher global distress and anxiety than those insufficiently active $(p=0.01$ and $p<0.05$, respectively) (Table 2). There were no differences for depression $(p=0.09)$ or somatization $(p=0.06)$. Inactive men had higher supportive care needs for physical and daily living compared with those sufficiently and insufficiently active $(p<0.05)$ and higher need for care and support when compared with those insufficiently active $(p<0.05)$. Inactive men also had higher need for sexuality and information needs than sufficiently active men $(p<0.05)$. Total supportive care need score was higher in inactive men than those insufficiently and sufficiently active men $(p<0.05)$.

\section{Disease-specific and health-related quality of life}

Prostate cancer-specific QoL is shown in Table 3. Inactive men had a higher IPSS score than insufficient $(p<0.01)$ and sufficiently active men $(p<0.05)$. Differences were 
Table I. Sociodemographic and clinical variables in inactive, insufficiently active, and sufficiently active men

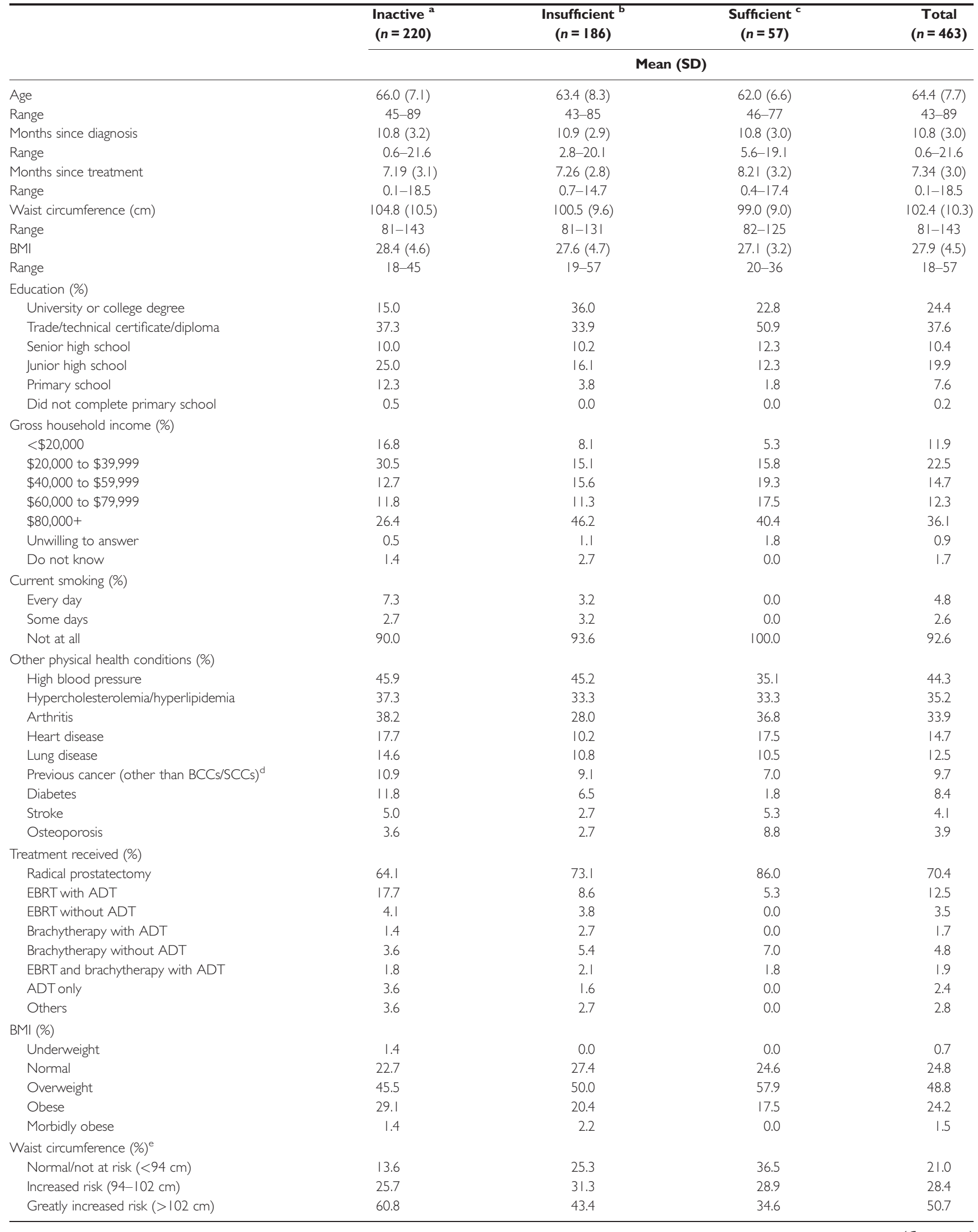


Table I. (Continued)

\begin{tabular}{|c|c|c|c|c|}
\hline & $\begin{array}{l}\text { Inactive }{ }^{a} \\
(n=220)\end{array}$ & $\begin{array}{c}\text { Insufficient }{ }^{b} \\
(n=186)\end{array}$ & $\begin{array}{l}\text { Sufficient }^{c} \\
(n=57)\end{array}$ & $\begin{array}{c}\text { Total } \\
(n=463)\end{array}$ \\
\hline & \multicolumn{4}{|c|}{ Mean (SD) } \\
\hline Pre-contemplation & 6.8 & 1.6 & 0.0 & 3.9 \\
\hline Contemplation & 17.3 & 3.8 & 0.0 & 9.7 \\
\hline Preparation & 27.7 & 14.5 & 1.8 & 19.2 \\
\hline Unable to be assigned & 2.7 & 1.6 & 1.8 & 2.2 \\
\hline
\end{tabular}

EBRT, external beam radiation; ADT, androgen deprivation therapy.

anactive, no moderate or strenuous exercise;

'Insufficient, insufficiently active;

'Sufficient, sufficiently active;

'Basal Cell Carcinomas/Squamous Cell Carcinoma;

$e_{n}=45$.

Table 2. Psychological distress and supportive care needs in inactive, insufficiently active, and sufficiently active men

\begin{tabular}{|c|c|c|c|c|}
\hline & Inactive $^{\text {a }}$ & Insufficient ${ }^{b}$ & Sufficient $^{c}$ & Total \\
\hline & \multicolumn{4}{|c|}{ Mean (SD) } \\
\hline \multicolumn{5}{|l|}{$\mathrm{BSI}-18^{\mathrm{d}}$} \\
\hline Depression & $1.55(3.06)$ & $1.08(1.82)$ & $1.28(1.93)$ & $1.33(2.5 \mathrm{I})$ \\
\hline Anxiety & $1.57(2.56)$ & $1.14 *(1.83)$ & $1.37(1.79)$ & $1.37(2.21)$ \\
\hline Global distress & $4.67(6.91)$ & $3.28 *(4.14)$ & $3.84(4.47)$ & $4.01(5.69)$ \\
\hline Psychological & $|5.2|(7.4 \mid)$ & | $4.64(6.58)$ & $14.89(6.84)$ & | $4.94(7.0 \mid)$ \\
\hline Health system/information & $19.66(7.55)$ & $18.12(6.28)$ & 16.84*(5.03) & I8.69 (6.84) \\
\hline Patient care & $7.95(3.31)$ & $7.12 *(2.24)$ & $7.05(2.40)$ & $7.51(2.84)$ \\
\hline Sexuality & $10.42(4.68)$ & $10.08(4.24)$ & $9.29 *(3.90)$ & $10.15(4.42)$ \\
\hline Total & $65.62(21.30)$ & $61.15 *(17.59)$ & $58.73 *(16.12)$ & $62.97(19.43)$ \\
\hline
\end{tabular}

BSI-18, Brief Symptom Inventory-18; SCNS-SF34, Supportive Care Needs Survey Short Form-34.

anactive, no moderate or strenuous exercise;

'Insufficient, insufficiently active;

'Sufficient, sufficiently active;

${ }^{\mathrm{d} H i g h e r}$ scores indicate higher distress or higher supportive care needs;

eSCNS-SF34 scored by summing items in each domain: physical (8 items; range 8-34); psychological (10 items; range 10-49); health system/information (II items; range II-50); patient care (5 items; range 5-24); sexuality (5 items; range 5-25); total (39 items; range 39-154).

${ }^{*} p<0.05$ compared with inactive;

*k $p<0.01$ compared with inactive.

also detected among various EPIC dimensions and subscales. For example, inactive men had lower urinary bother scores compared with insufficiently and sufficiently active men $(p<0.05)$. Urinary dimension and urinary function scores were lower in inactive compared with those in insufficiently active men $(p<0.01)$. Sexual dimension and sexual function scores were lower in the inactive compared with those of insufficiently active $(p<0.001)$ and sufficiently active $(p=0.01)$ men, and sexual bother scores lower compared with those of the insufficiently active men $(p<0.01)$. There were no differences for any of the remaining EPIC domains or subscales. Health-related QoL outcomes are shown in Table 4. Inactive men had lower AQoL-8D utility scores than the insufficiently active men $(p<0.01)$, lower independent living scores than insufficiently active $(p=0.01)$ and the sufficiently active men $(p<0.05)$, lower coping scores than insufficiently active $(p<0.01)$ and sufficiently active men $(p<0.01)$, and lower senses $(p<0.01)$ and physical superdimension scores $(p<0.05)$ than insufficiently active men.

\section{Discussion}

The present study reports four important findings: (1) only $12 \%$ of this population-based sample of Australian men with prostate cancer reported meeting current exerciseoncology guidelines with the large majority ( $48 \%$ ) inactive (not undertaking any moderate or vigorous exercise); (2) $\sim 30 \%$ of men were at least in the contemplation and preparation stages of changes suggesting presence of a teachable 
Table 3. Disease-specific QoL in inactive, insufficiently active, and sufficiently active men

\begin{tabular}{|c|c|c|c|c|}
\hline & Inactive $^{\mathbf{a}}$ & Insufficient $^{\mathbf{b}}$ & Sufficient $^{c}$ & Total \\
\hline & \multicolumn{4}{|c|}{ Mean (SD) } \\
\hline IPSS $^{d}$ & $8.45(6.79)$ & 6.19** (5.86) & $5.74 *(5.01)$ & $7.21(6.33)$ \\
\hline \multicolumn{5}{|l|}{ EPIC $^{\mathrm{e}}$} \\
\hline Urinary & $83.75(16.43)$ & $88.37 * *$ (13.65) & $88.92(13.25)$ & $86.26(15.14)$ \\
\hline Function & $84.04(17.55)$ & $89.14 * *$ (I5.02) & $88.15(15.55)$ & $86.60(16.48)$ \\
\hline Bother & $83.51(17.93)$ & $87.83 *(14.77)$ & $89.47 *(13.03)$ & $85.98(|6.3|)$ \\
\hline Bother & $93.21(13.80)$ & $94.76(11.66)$ & $95.68(7.95)$ & $94.14(12.38)$ \\
\hline Sexual & $30.91(21.16)$ & $42.30 * * * *(23.17)$ & $44.26 * *(25.42)$ & $37.14(23.26)$ \\
\hline Function & $20.7 \mid$ (22.88) & $32.97 * * * *(26.88)$ & $36.10 * * *(27.32)$ & $27.55(25.91)$ \\
\hline Bother & $54.06(36.65)$ & $63.27 * * * 30.47)$ & 62.61 (33.58) & $58.81(34.14)$ \\
\hline Hormonal $(n=82)$ & $82.08(15.15)$ & $80.16(9.74)$ & $76.7 \mid(11.04)$ & 81.21 (13.42) \\
\hline Function & $76.64(17.65)$ & $71.73(12.96)$ & $70.00(10.80)$ & $74.76(16.08)$ \\
\hline
\end{tabular}

IPSS, International Prostate Symptom Score; EPIC, Expanded Prostate Index Composite.

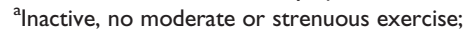

'Insufficient, insufficiently active;

'Sufficient, sufficiently active; ${ }^{\mathrm{d}}$ Higher scores indicate greater symptom severity;

eHigher scores indicate better QoL.

$* p<0.05$ compared with inactive;

*** $p<0.01$ compared with inactive;

$* k * k p<0.001$ compared with inactive.

Table 4. Health-related QoL in inactive, insufficiently active, and sufficiently active men and Australian population norms [3।]

\begin{tabular}{|c|c|c|c|c|c|}
\hline & Inactive $^{\mathbf{a}}$ & Insufficient $^{\text {b }}$ & Sufficient $^{c}$ & Total & Australian norms - male \\
\hline & \multicolumn{4}{|c|}{ Mean (SD) } & Mean (SE) \\
\hline \multicolumn{6}{|l|}{$\mathrm{AQoL}^{\mathrm{d}}$} \\
\hline Independent Living & $0.88(0.14)$ & $0.92 *(0.11)$ & $0.94 *(0.10)$ & $0.90(0.13)$ & $0.95(0.003)$ \\
\hline Happiness & $0.82(0.12)$ & $0.84(0.10)$ & $0.85(0.10)$ & $0.83(0.11)$ & $0.82(0.004)$ \\
\hline Mental Health & $0.70(0.15)$ & $0.73(0.13)$ & $0.73(0.15)$ & $0.72(0.14)$ & $0.71(0.005)$ \\
\hline Self-worth & $0.88(0.14)$ & $0.90(0.10)$ & $0.90(0.11)$ & $0.89(0.12)$ & $0.89(0.004)$ \\
\hline Pain & $0.82(0.21)$ & $0.86(0.20)$ & $0.86(0.18)$ & $0.84(0.20)$ & $0.91(0.004)$ \\
\hline Senses & $0.83(0.12)$ & $0.87 * * * 10.10)$ & $0.87(0.10)$ & $0.85(0.11)$ & $0.90(0.004)$ \\
\hline Mental & $0.52(0.21)$ & $0.56(0.18)$ & $0.57(0.20)$ & $0.54(0.20)$ & $0.5 \mid(0.007)$ \\
\hline Physical & $0.72(0.19)$ & $0.79 *(0.18)$ & $0.79(0.17)$ & $0.76(0.19)$ & $0.85(0.005)$ \\
\hline
\end{tabular}

anactive, no moderate or strenuous exercise;

'Insufficient, insufficiently active;

'Sufficient, sufficiently active;

${ }^{\mathrm{d}}$ Higher scores indicate better QoL.

${ }^{*} p<0.05$ compared with inactive;

$* * k p<0.01$ compared with inactive.

moment; (3) psychological distress differed in relation to activity levels with inactive prostate cancer survivors reporting higher global distress and anxiety; and (4) unmet supportive care needs were higher and several aspects of health-related and disease-specific QoL lower in inactive men including domains of urinary and sexual function.

To our knowledge, this is the first population-based study to examine adherence to contemporary exercise-oncology recommendations including aerobic and resistance modes in men with prostate cancer who have received or were receiving curative therapy. We found that very few prostate cancer survivors $(\sim 12 \%)$ met exercise recommendations with $\sim 40 \%$ insufficiently active and a large proportion inactive $(\sim 48 \%)$. Previous reports from North America on aerobic exercise recommendations in cancer survivors at least one year post-diagnosis suggested higher prevalence of participation at $\sim 43 \%$ of prostate cancer survivors [23] and more recently $\sim 47 \%$ in middle-aged cancer survivors (including a variety of cancers) from the Behavior Risk Factor Surveillance System [24]. These reports were either initiated or 
published prior to the more recent recommendations from the American Cancer Society [12] and American College of Sports Medicine [13] that include integration of resistance exercise. Compliance to aerobic-only exercise guidelines from our total cohort using the same self-reported instrument to assess physical activity was $\sim 33 \%$, which is lower than those previously reported in North America ( 43\%) [23].

Extensive evidence has accumulated in recent years on the benefits of aerobic and resistance trainings in men with prostate cancer during active therapy or in those who have completed therapy including radiation/androgen deprivation [1,2,4,6-8]. Vigorous exercise has been also associated with reduction in prostate cancer-specific death by $\sim 60 \%$ in a cohort of 2705 men with prostate cancer from the Health Professionals Follow-up Study [9]. Recent expert reviews in urology/oncology have further incorporated aerobic and resistance exercise interventions as evidence-based strategies to mitigate toxicities from androgen deprivation including components of metabolic syndrome, sexual dysfunction, and fatigue [25]. With $88 \%$ of our Australian cohort of men not adhering to contemporary exercise-oncology recommendations, strategies are urgently required to increase prostate cancer survivors' participation in aerobic and resistance exercise programs. For example, we recently reported a year-long exercise trial in prostate cancer survivors with both supervised and home-based components that may facilitate translation into practice and improve participation [6].

Our early study in supportive care needs for men with prostate cancer indicated that one-third of men reported a moderate to high need for help in the sexuality, psychological, and health system and information domains [17], a finding confirmed elsewhere [26]. Crucially, in this study, inactive men had higher supportive care needs compared with men who were insufficiently and sufficiently active. In addition, although the prevalence of distress in this population was low [27], men who were inactive had higher anxiety and global distress compared with those who were insufficiently active. The link between inactivity and psychological distress suggests that exercise interventions have potential to provide health benefits beyond physical functioning.

Enhanced continence post-surgery has been reported in prostate cancer survivors who have normal weight and are physically active compared with survivors who are obese and sedentary. Further, 58 weeks post-surgery, the incidence of incontinence appears to be the same for overweight but physically active men compared with normal weight but sedentary men [28]. Our cohort of inactive men had a higher IPSS score based on urinary symptoms than those insufficiently and sufficiently active men. Notably, the majority of our cohort $\sim 70 \%$ underwent radical prostatectomy hence supporting that meeting specific exercise levels may lead to better continence. We also noted that our cohort of inactive men had greater mean waist circumference (i.e., $\sim 105 \mathrm{~cm}$ ) compared with insufficiently (i.e., $101 \mathrm{~cm}$ ) and sufficiently (i.e., $98 \mathrm{~cm}$ ) active men. Importantly, waist circumference $\geq 102 \mathrm{~cm}$ is a key criterion for metabolic syndrome diagnosis [29]. Further, abdominal obesity, such as waist circumference, correlates with fat mass changes by dual-energy X-ray absorptiometry and has been extensively reported in large epidemiological studies on cardiovascular and metabolic diseases [30]. Increasing physical activity is a key strategy for reducing fat mass and is more effective when combined with caloric restriction, but such an energy imbalance also results in loss of muscle and bone mass unless accompanied by resistance training. This further emphasizes the importance of cancer survivors meeting both the aerobic and resistance training components of the guidelines.

We further identified levels of activity in relation to disease-specific and health-related QoL. Sexual dimension and sexual function scores were lower in the inactive compared with insufficiently active and sufficiently active men, and sexual bother scores lower compared with the insufficiently active men. There was a significant and clinically meaningful advantage for the insufficiently active men over the inactive men for several dimensions of QoL. However, the sufficiently active men exhibited equal or only marginally better scores compared with those insufficiently active. It appears that being inactive has considerable negative consequences for QoL, and even a modest amount of moderate/vigorous activity is associated with benefit across a wide range of QoL dimensions. If the men were not inactive, then their scores were actually equal to or higher than the Australian norms [31] for all of the QoL dimensions except pain, senses, and physical superdimension.

Our study has several features that are worthy of comment. First, we used a large population-based cohort of metropolitan and rural Australian prostate cancer survivors, and this is the first report on adherence to contemporary exercise-oncology recommendations including aerobic and resistance exercise modes. Second, we targeted a well-defined population of men with prostate cancer who received or were undergoing active therapy. Third, we employed a range of well-established instruments of distress, unmet supportive care needs, and disease-specific and health-related QoL in relation to adherence to current exercise-oncology guidelines. Limitations included the following: the cross-sectional nature of the study that does not permit us to infer causality; the leisure-time assessment does not include occupational or domestic work and is self-assessed, which is normally inferior to objective measures (e.g., accelerometer). However, it was not feasible to implement objective measures of physical activity given the large number of participants involved in the study.

In summary, approximately $88 \%$ of prostate cancer survivors in Australia do not meet physical activity guidelines for people with cancer, and almost half is totally inactive. Lack of physical activity appears to contribute to higher 
psychological distress, greater reported unmet supportive care needs, and poorer QoL. However, most men were at least contemplating behavioral change, and of those who were inactive, almost a third were preparing for change suggesting an opportunity for intervention capitalizing on a teachable moment. Further research addressing effective and translatable lifestyle interventions for this patient group are needed.

\section{Acknowledgements}

This project was funded by Cancer Australia and beyondblue (ID APP1008320). S. K. C. had full access to all the data in the study and takes responsibility for the integrity of the data and the accuracy of the data analysis. The sponsors did not participate in the design or conduct of the study; collection, management, analysis, and interpretation of the data; or in the preparation, review, or approval of the manuscript. All authors had no conflict of interest, including relevant financial interests, activities, relationships, and affiliations to declare relating to this manuscript. D. A. G. is funded by a Movember New Directions Development Award from Prostate Cancer Foundation of Australia and Cancer Council Western Australia Research Fellowship. S. K. C. is an Australian Research Council Future Fellow, and A. G. is funded by a Cancer Institute NSW grant. We gratefully acknowledge the support of the Urological Society of Australia and New Zealand; of Mr Bill McHugh, Mr Spence Broughton and Mr Peter Dornan as consumer advisors; and of Ms Brigid Hanley and Ms Sylvia Burns as prostate cancer nurse advisors in the undertaking of this research.

\section{References}

1. Segal RJ, Reid RD, Courneya KS, et al. Resistance exercise in men receiving androgen deprivation therapy for prostate cancer. J Clin Oncol 2003;21:1653-1659.

2. Segal RJ, Reid RD, Courneya KS, et al. Randomized controlled trial of resistance or aerobic exercise in men receiving radiation therapy for prostate cancer. $J$ Clin Oncol 2009;27:344-351.

3. Galvao DA, Nosaka K, Taaffe DR, et al. Resistance training and reduction of treatment side effects in prostate cancer patients. Med Sci Sports Exerc 2006;38:2045-2052.

4. Galvao DA, Taaffe DR, Spry N, Joseph D, Newton RU. Combined resistance and aerobic exercise program reverses muscle loss in men undergoing androgen suppression therapy for prostate cancer without bone metastases: a randomized controlled trial. J Clin Oncol 2010;28:340-347.

5. Beydoun N, Bucci JA, Chin YS, Spry N, Newton R, Galvao DA. Prospective study of exercise intervention in prostate cancer patients on androgen deprivation therapy. $J$ Med Imaging Radiat Oncol 2014;58:369-376.

6. Galvao DA, Spry N, Denham J, et al. A multicentre year-long randomised controlled trial of exercise training targeting physical functioning in men with prostate cancer previously treated with androgen suppression and radiation from TROG 03.04 RADAR. Eur Urol 2014;65:856-864.

7. Bourke L, Gilbert S, Hooper R, et al. Lifestyle changes for improving disease-specific quality of life in sedentary men on long-term androgen-deprivation therapy for advanced prostate cancer: a randomised controlled trial. Eur Urol 2014;65:865-872.

8. Cormie P, Galvao DA, Spry N, et al. Can supervised exercise prevent treatment toxicity in prostate cancer patients initiating androgen deprivation therapy: a randomised controlled trial. BJU Int 2014; Advance online publication; doi: $10.1111 /$ bju. 12646
9. Kenfield SA, Stampfer MJ, Giovannucci E, Chan JM. Physical activity and survival after prostate cancer diagnosis in the health professionals follow-up study. J Clin Oncol 2011; 29:726-732.

10. Holmes MD, Chen WY, Feskanich D, Kroenke CH, Colditz GA. Physical activity and survival after breast cancer diagnosis. JAMA 2005;293:2479-2486.

11. Meyerhardt JA, Giovannucci EL, Holmes $\mathrm{MD}$, et al. Physical activity and survival after colorectal cancer diagnosis. J Clin Oncol 2006;24:3527-3534.

12. Rock CL, Doyle C, Demark-Wahnefried W, et al. Nutrition and physical activity guidelines for cancer survivors. CA Cancer J Clin 2012;62:243-274.

13. Schmitz KH, Courneya KS, Matthews C, et al. American College of Sports Medicine roundtable on exercise guidelines for cancer survivors. Med Sci Sports Exerc 2010;42: 1409-1426.

14. Chambers SK, Newton RU, Girgis A, et al. Living with prostate cancer: randomised controlled trial of a multimodal supportive care intervention for men with prostate cancer. BMC Cancer 2011;11:317.

15. Godin G, Shephard RJ. A simple method to assess exercise behavior in the community. Can J Appl Sport Sci 1985;10:141-146.

16. Derogatis LR, Lopez MC. Brief Symptom Inventory 18: Administration, Scoring and Procedures Manual, National Computer Systems, Inc.: Minneapolis, 2000.

17. Steginga SK, Occhipinti S, Dunn J, Gardiner RA, Heathcote P, Yaxley J. The supportive care needs of men with prostate cancer (2000). Psycho-Oncology 2001;10:66-75.

18. Richardson J, Khan M, Iezzi A, et al. The AQoL-8D (PsyQoL) MAU Instrument: Overview (Research Paper 39), Centre for Health Economics, Monash University: Melbourne, 2009.

19. Barry MJ, Fowler FJ Jr, O’Leary MP, et al. The American Urological Association Symptom Index for benign prostatic hyperplasia. The Measurement Committee of the
American Urological Association. J Urol 1992;148:1549-1557.

20. Litwin MS, Hays RD, Fink A, Ganz PA, Leake B, Brook RH. The UCLA Prostate Cancer Index: development, reliability, and validity of a health-related quality of life measure. Med Care 1998;36:1002-1012.

21. Wei JT, Dunn RL, Litwin MS, Sandler HM, Sanda MG. Development and validation of the Expanded Prostate Cancer Index Composite (EPIC) for comprehensive assessment of health-related quality of life in men with prostate cancer. Urology 2000;56:899-905.

22. Clark MM, Novotny PJ, Patten CA, et al. Motivational readiness for physical activity and quality of life in long-term lung cancer survivors. Lung Cancer 2008;61(1):117-122.

23. Blanchard CM, Courneya KS, Stein K. Cancer survivors' adherence to lifestyle behavior recommendations and associations with health-related quality of life: results from the American Cancer Society's SCS-II. J Clin Oncol 2008;26(13):2198-2204.

24. Nayak P, Holmes HM, Nguyen HT, Elting LS. Self-reported physical activity among middle-aged cancer survivors in the United States: behavioral risk factor surveillance system survey, 2009. Prev Chronic Dis 2014;11: E156.

25. Nguyen PL, Alibhai SM, Basaria S, et al. Adverse effects of androgen deprivation therapy and strategies to mitigate them. Eur Urol 2014; Advance online publication; doi: 10.1016/j.eururo.2014.07.010.

26. Smith DP, Supramaniam R, King MT, Ward J, Berry M, Armstrong BK. Age, health, and education determine supportive care needs of men younger than 70 years with prostate cancer. J Clin Oncol 2007;25: 2560-2566.

27. Chambers SK, Zajdlewicz L, Youlden DR, Holland JC, Dunn J. The validity of the distress thermometer in prostate cancer populations. Psycho-Oncology 2014;23(2): 195-203.

28. Wolin KY, Luly J, Sutcliffe S, Andriole GL, Kibel AS. Risk of urinary incontinence 
following prostatectomy: the role of physical activity and obesity. J Urol 2010;183: 629-633.

29. Executive Summary of the Third Report of the National Cholesterol Education Program (NCEP) Expert Panel on Detection, Evaluation, and Treatment of High Blood Cholesterol in Adults (Adult Treatment Panel III). JAMA 2001;285:2486-2497.

30. See R, Abdullah SM, McGuire DK, et al. The association of differing measures of overweight and obesity with prevalent atherosclerosis: the Dallas Heart Study. J Am Coll Cardiol 2007;50:752-759.
31. Richardson J, Khan MA, Chen G, Iezzi A, Maxwell A. Population norms and Australian profile using the assessment of quality of life (AQoL) 8D utility instrument (research paper 72). Melbourne, Centre for Health Economics, Monash University, 2012 